BMJ Open

Sport \&

Exercise

Medicine

\title{
Validity of the Fitbit activity tracker for measuring steps in community-dwelling older adults
}

\author{
Serene S Paul, ${ }^{1}$ Anne Tiedemann, ${ }^{1}$ Leanne M Hassett, ${ }^{1,2}$ Elisabeth Ramsay, ${ }^{1}$ \\ Catherine Kirkham, ${ }^{1}$ Sakina Chagpar, ${ }^{1}$ Catherine Sherrington ${ }^{1}$
}

To cite: Paul SS,

Tiedemann A, Hassett LM, et al. Validity of the Fitbit activity tracker for measuring steps in community-dwelling older adults. BMJ Open Sport Exerc Med 2015;0:e000013. doi:10.1136/bmjsem-2015000013

- Prepublication history for this paper is available online To view these files please visit the journal online (http://dx.doi.org/10.1136/ bmjsem-2015-000013).

Accepted 4 June 2015

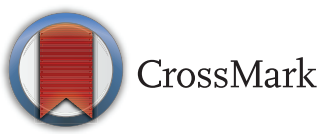

${ }^{1}$ The George Institute for Global Health, Sydney Medical School, The University of Sydney, Sydney, New South Wales, Australia ${ }^{2}$ Faculty of Health Sciences, The University of Sydney, Sydney, New South Wales, Australia

Correspondence to

Dr Serene S Paul;

spaul@georgeinstitute.org.au

\section{ABSTRACT}

Background: Commercially available activity monitors, such as the Fitbit, may encourage physical activity. However, the accuracy of the Fitbit in older adults remains unknown. This study aimed to determine (1) the criterion validity of Fitbit step counts compared to visual count and ActiGraph accelerometer step counts and (2) the accuracy of ActiGraph step counts compared to visual count in community-dwelling older people.

Methods: Thirty-two community-dwelling adults aged over 60 wore Fitbit and ActiGraph devices simultaneously during a 2 min walk test (2MWT) and then during waking hours over a 7-day period. A physiotherapist counted the steps taken during the 2MWT.

Results: There was excellent agreement between Fitbit and visually counted steps (intraclass correlation coefficient $\left(\mathrm{ICC}_{2,1}\right)=0.88,95 \% \mathrm{Cl} 0.76$ to 0.94$)$ from the 2MWT, and good agreement between Fitbit and ActiGraph $\left(\mathrm{ICC}_{2,1}=0.66,95 \% \mathrm{Cl} 0.41\right.$ to 0.82 ), and between ActiGraph and visually counted steps $\left(\mathrm{ICC}_{2,1}=0.60,95 \% \mathrm{Cl} 0.33\right.$ to 0.79$)$. There was excellent agreement between the Fitbit and ActiGraph in average steps/day over 7 days $\left(\mathrm{ICC}_{2,1}=0.94,95 \% \mathrm{Cl}\right.$ 0.88 to 0.97 ). Percentage agreement was closest for Fitbit steps compared to visual count (mean 0\%, SD $4 \%$ ) and least for Fitbit average steps/day compared to the ActiGraph (mean 13\%, SD 25\%).

Conclusions: The Fitbit accurately tracked steps during the 2MWT, but the ActiGraph appeared to underestimate steps. There was strong agreement between Fitbit and ActiGraph counted steps. The Fitbit tracker is sufficiently accurate to be used among community-dwelling older adults to monitor and give feedback on step counts.

\section{BACKGROUND}

Physical inactivity is a major risk factor for many chronic conditions, and contributes to early mortality ${ }^{1}$ and rising healthcare costs. ${ }^{2}$ Although prevalent throughout the lifespan, physical inactivity and sedentary behaviour (time spent sitting and lying down) are particularly common among older adults. ${ }^{3}$

\section{What are the new findings}

- The Fitbit activity tracker is able to accurately quantify steps in community-dwelling older adults.

- The ActiGraph accelerometer undercounts steps in older adults with overall good mobility.

- The Fitbit tracker is sufficiently accurate to be used among community-dwelling older adults to monitor and give feedback on step counts.

Health problems that are more common in older age may also contribute to low physical activity levels. ${ }^{4}$

Moderate to vigorous physical activity is known to induce health benefits. ${ }^{5}$ There is mounting evidence that large amounts of sedentary behaviour is harmful to health even in those who also engage in physical activity. $^{6}$ Current guidelines recommend older adults engage in at least $150 \mathrm{~min}$ of moderate to vigorous physical activity per week, in bouts lasting for $10 \mathrm{~min}$ or more, as well as minimise sedentary behaviours. ${ }^{3} 7$ Increasing the overall number of steps taken daily can enable people to increase their amount of moderate to vigorous physical activity $^{5}$ in addition to increasing light intensity activity and reducing sedentary behaviour. ${ }^{8}$ Increasing the amount of steps taken daily may, therefore, be one method of enabling older adults to increase their overall physical activity levels.

Pedometers quantify and give feedback on physical activity and this increases physical activity levels. ${ }^{9}$ The Fitbit tracker (Fitbit Inc, San Francisco, California, USA) is a relatively affordable commercially-available pedometer that automatically records step counts and can provide instant feedback on either the device itself or via simple software accessed via the internet. Fitbit trackers come in a variety of small unobtrusive 
activity monitors that can be clipped onto a belt, attached to clothing or worn around the wrist. The Fitbit's internet and smart phone/tablet interfaces also allow physical activity levels for each individual user to be tracked longitudinally, which can enable the user to monitor their activity trends over time and could also allow healthcare professionals to tailor physical activity recommendations for individuals, thereby encouraging maintenance of physical activity behaviour.

Recent validation studies have demonstrated that the Fitbit is accurate in tracking steps in young ${ }^{10}{ }^{11}$ and middle-aged $^{12}$ adults; however, no study to date has investigated the accuracy of the Fitbit for tracking physical activity in older adults. Older adults frequently present with a wide range of physical disabilities and evidence suggests that activity monitors are less accurate in measuring activity in people who walk with slower gait speeds, ${ }^{13}$ use walking aids ${ }^{14}$ or have gait impairments. ${ }^{15} 16$ Determining the accuracy of commercially available activity monitors, such as the Fitbit tracker, for use in older adults would assist health professionals to decide if these devices are appropriate for use by their older clients. ${ }^{17}$ This study, therefore, aimed to determine the criterion validity ${ }^{18}$ (ie, accuracy) of a Fitbit tracker's (One or Zip) step count compared to visual count by a health professional and to the well-validated ActiGraph GT3X+ accelerometer ${ }^{19}$ (ActiGraph Corp, Pensacola, Florida, USA) in community-dwelling older adults. A secondary aim was to determine the accuracy of ActiGraph accelerometer step counts compared to visual count in this population.

\section{METHODS}

\section{Participants}

Participants were community-dwelling older adults from Sydney, Australia, who were randomised to the intervention group of a trial investigating the effect of a behaviour change programme that aimed to increase physical activity participation and reduce fall risk. ${ }^{20}$ Participants were aged over 60 years, lived at home, were regular (weekly) users of the internet via a computer or tablet device and left their house regularly (at least once per week) without physical assistance from another person. Individuals were excluded if they: were housebound (ie, had not gone outside without physical assistance from another person in the past month); had a cognitive impairment (a diagnosis of dementia or a Memory Impairment Screen score $<5 ;{ }^{21}$ had insufficient English language skills to fully participate in the programme; had a progressive neurological condition (eg, Parkinson's disease) or a medical condition precluding exercise (eg, unstable cardiac disease); were currently participating in $\geq 150 \mathrm{~min}$ of moderate intensity physical activity per week and had undergone a fall risk assessment in the past year with subsequent adoption of recommendations.

\section{Procedures}

Participants wore a Fitbit tracker (One or Zip) simultaneously with an ActiGraph GT3X+ accelerometer on their right hip. Each device was programmed with the participant's age and gender, the Fitbit was programmed with the participant's height and the ActiGraph was programmed with the participant's weight. The ActiGraph collected raw data at $30 \mathrm{~Hz}$.

Participants performed a 2 min walk test (2MWT) in the space available in their homes (usually a corridor approximately $7-10 \mathrm{~m}$ in length or a circuit of approximately $15 \mathrm{~m}$ ), during which a research physiotherapist also observed and counted (with a hand-held stationery counter) the number of steps the participant took. Participants were instructed to stand still for $10 \mathrm{~s}$ prior to and after the 2MWT, and the start and finish times of the 2MWT were recorded. The number of steps recorded by the Fitbit tracker was calculated as the difference between the step count displayed by the tracker at the start and at the end of the 2MWT. The number of steps recorded by the ActiGraph during the 2MWT was extracted in $1 \mathrm{~s}$ epochs with ActiLife 6 software.

Participants also wore the Fitbit simultaneously with the ActiGraph accelerometer during waking hours (except for water sports or bathing) for a 7-day period. Participants completed a physical activity log for the week-long period. Fitbit tracker and ActiGraph accelerometer data were checked against participants' activity logs for obvious inconsistencies and any erroneous data were removed. Fitbit tracker data were extracted from the internet interface as the number of steps taken on each day, calculated using Fitbit's proprietary algorithm and participants' anthropometry. Daily step counts were extracted from the ActiGraphs in $60 \mathrm{~s}$ epochs using the Freedson Adult (1998) equation without any wear time validation as this process is not available with the Fitbit trackers. Step counts from the Fitbit and ActiGraph were averaged over the 7-day period for analysis.

\section{Data analysis}

Intraclass correlation coefficients $\left(\mathrm{ICC}_{2,1}\right)$ were used to examine agreement between step counts taken from the Fitbit, ActiGraph and by the physiotherapist. An ICC $\geq 0.75$ was considered excellent, 0.60-0.74 good, 0.40-0.59 fair and $<0.40$ poor. ${ }^{22}$ Percentage agreement for step counts against the criterion measure of visual count, or of the ActiGraph for Fitbit versus ActiGraph comparisons, were also calculated. Bland-Altman plots were used to visualise any systematic differences between step counts from the Fitbit, ActiGraph and visual count. Data were analysed using SPSS V.22 (IBM Corporation, Armonk, New York, USA).

\section{RESULTS}

Thirty-two individuals (12 male, 20 female) participated in this validation study. Demographic and health characteristics of the sample are shown in table 1 . 
Table 1 Participant characteristics for the sample $(\mathrm{N}=32)$

\begin{tabular}{ll}
\hline Characteristic & Mean (SD) or N (\%) \\
\hline Age (years) & $67.7(5.7)$ \\
Gender (male; female) & $12(38 \%) ; 20(63 \%)$ \\
Memory impairment screen (0-8) & $7.6(0.9)$ \\
Number of comorbidities & $3.3(2.4)$ \\
0 & $2(6 \%)$ \\
$1-2$ & $13(41 \%)$ \\
$3-5$ & $11(34 \%)$ \\
$6+$ & $6(19 \%)$ \\
Reported falling in the past year & $10(31 \%)$ \\
2 min walk test (steps)* & $245.1(18.4)$ \\
Uses a walking aid & 0 \\
$\quad$ Indoors & $1(3 \%)$ \\
Outdoors & \\
Living situation & $12(37 \%)$ \\
$\quad$ Lives alone & $15(47 \%)$ \\
$\quad$ Lives with spouse \pm children & $5(16 \%)$ \\
$\quad$ Lives with friend/relative &
\end{tabular}

Step counts for the 2MWT measured with the Fitbit tracker showed excellent agreement with visual count by a physiotherapist $\left(\mathrm{ICC}_{2,1}=0.88,95 \%\right.$ CI 0.76 to 0.94$)$. There was good agreement between Fitbit and ActiGraph counted steps from the 2MWT $\left(\mathrm{ICC}_{2,1}=0.66,95 \%\right.$ CI 0.41 to 0.82 ), and between ActiGraph and visually counted steps $\left(\mathrm{ICC}_{2,1}=0.60,95 \%\right.$ CI 0.33 to 0.79$)$. Step counts recorded with the Fitbit during the 2MWT were on average 1.3 steps (95\% CI -4.7 to 2.1$)$ less than visual count, but 12.3 steps (95\% CI 4.3 to 20.2) more than the steps recorded by the ActiGraph for the 2MWT. Step counts recorded with the ActiGraph accelerometer were on average 13.5 steps (95\% CI -22.0 to -5.1 ) less than visual count recorded for the 2MWT. There was close percentage agreement for each device with a large proportion of participants achieving step counts within $5-15 \%$ of the criterion measure, with the Fitbit showing better percentage agreement to visual count than the ActiGraph (table 2). Bland-Altman plots revealed a bias by the ActiGraph for people who took fewer steps during the 2MWT (figure 1).

Average steps/day measured by the Fitbit tracker over the 7-day period showed excellent agreement with average steps/day measured by the ActiGraph accelerometer $\left(\mathrm{ICC}_{2,1}=0.94,95 \%\right.$ CI 0.88 to 0.97$)$. Daily step counts measured by the Fitbit over 7 days' wear were on average 716.7 steps per day (95\% CI 318.2 to 1115.1 ) more than daily steps measured by the ActiGraph. There was less percentage agreement between the Fitbit and ActiGraph for average daily steps with 34-66\% of participants having Fitbit scores within 5-15\% of ActiGraph scores (table 2). The Bland-Altman plot revealed no systematic bias in averaged daily step counts between the Fitbit tracker and ActiGraph accelerometer (figure 2).

\section{DISCUSSION}

This study found that in community-dwelling older adults, step counts measured by the Fitbit tracker strongly agreed with visually counted steps by a health professional, but the agreement with steps measured by the ActiGraph accelerometer was lower. Agreement was good between ActiGraph and visually counted steps. The ActiGraph appears to undercount steps in this cohort. This discrepancy was most apparent in people who took fewer steps during the 2MWT.

Our results extend the findings from other populations, including young adults, ${ }^{101123}$ middle-aged adults ${ }^{10} 12$ and

Table 2 Mean (SD) percentage agreement and number (\%) of participants with scores within $5 \%, 10 \%$ and $15 \%$ of the criterion measure for each comparison

\begin{tabular}{|c|c|c|c|c|}
\hline Comparison & $\begin{array}{l}\text { Percentage } \\
\text { agreement }\end{array}$ & $\begin{array}{l}5 \% \\
\text { agreement }\end{array}$ & $\begin{array}{l}10 \% \\
\text { agreement }\end{array}$ & $\begin{array}{l}15 \% \\
\text { agreement }\end{array}$ \\
\hline 2MWT Fitbit versus visual count & $0 \%(4 \%)$ & $27(84 \%)$ & $31(97 \%)$ & $32(100 \%)$ \\
\hline 2MWT Fitbit versus ActiGraph & $7 \%(16 \%)$ & $24(75 \%)$ & $27(84 \%)$ & $27(84 \%)$ \\
\hline 2MWT ActiGraph versus visual count & $6 \%(10 \%)$ & $22(69 \%)$ & $25(78 \%)$ & $28(88 \%)$ \\
\hline 7-day average steps/day Fitbit versus ActiGraph & $13 \%(25 \%)$ & $11(34 \%)$ & $16(50 \%)$ & $21(66 \%)$ \\
\hline
\end{tabular}

The latter measure in each comparison was used as the criterion measure.

2MWT, 2 min walk test. 

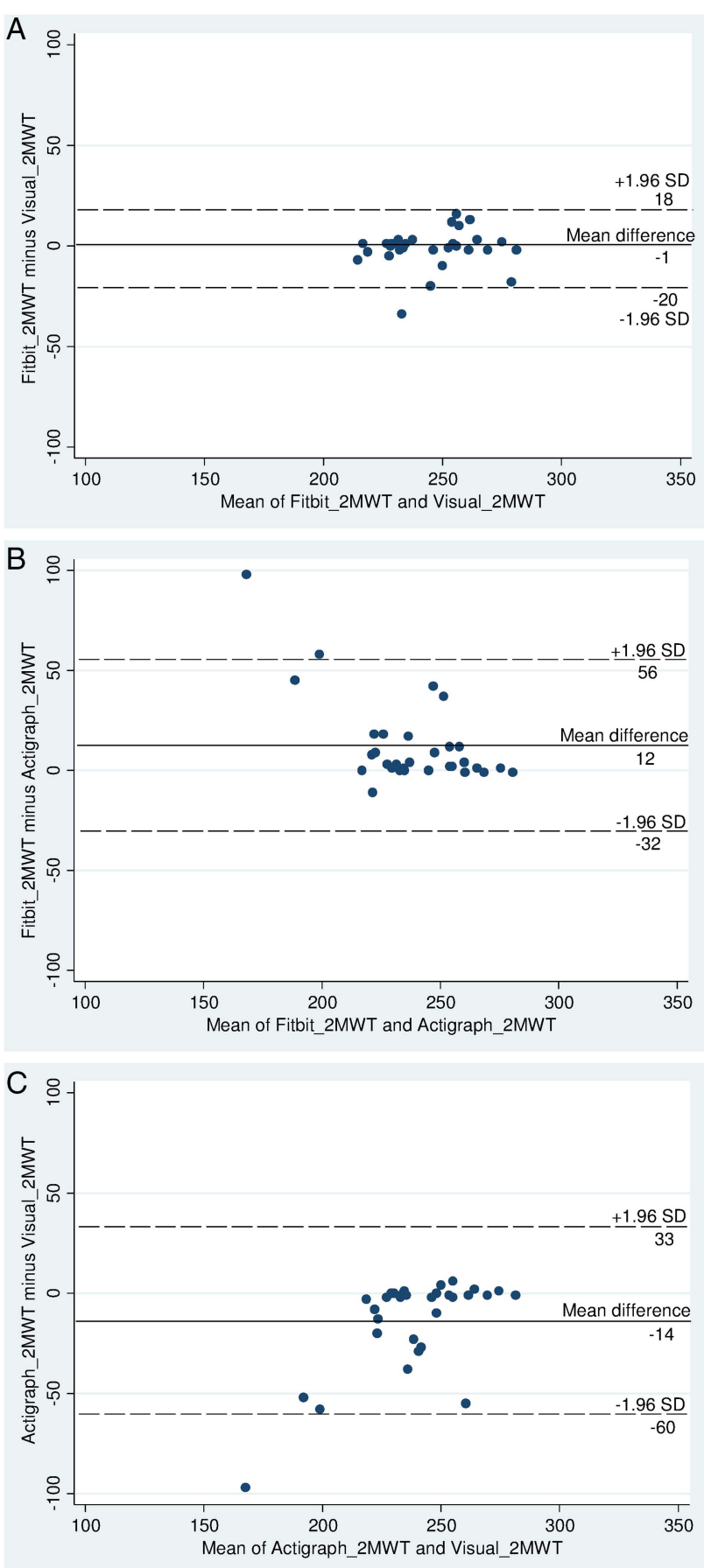

Figure 1 Bland-Altman plots comparing step counts over a 2 min walk test for the Fitbit tracker, ActiGraph accelerometer and visual count by a physiotherapist. (A) Comparison between the Fitbit tracker and visual count. (B) Comparison between the Fitbit tracker and ActiGraph accelerometer. (C) Comparison between the ActiGraph accelerometer and visual count. Actigraph_2MWT: ActiGraph step counts from the 2 min walk test. Fitbit_2MWT: Fitbit step counts from the 2 min walk test. Visual_2MWT: Physiotherapist-counted steps from the 2 min walk test. Solid line indicates the mean difference between the two measures, dashed lines indicate the limits of agreement (1.96 SDs of the mean difference).

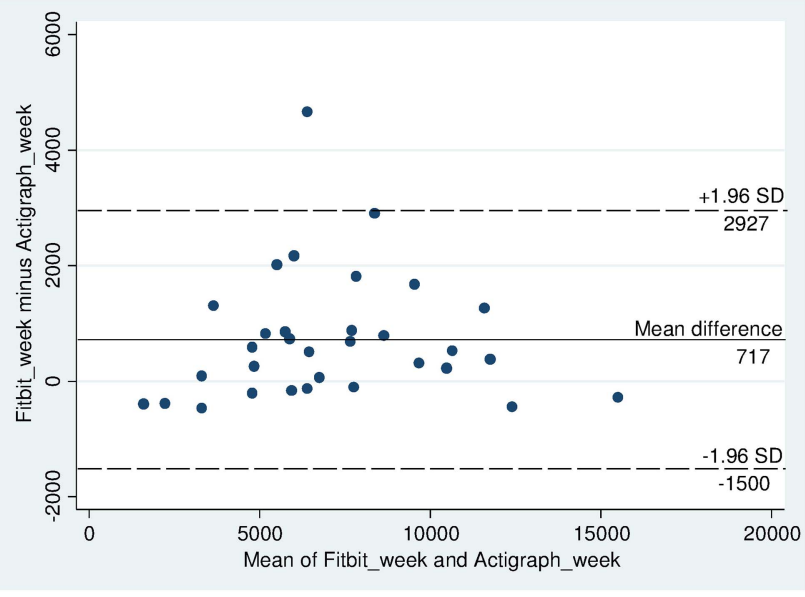

Figure 2 Bland-Altman plot comparing average steps/day over a 7-day period for the Fitbit tracker and ActiGraph accelerometer. Fitbit_week: Fitbit average steps/day from a 7-day period. Actigraph_week: ActiGraph average steps/day from a 7-day period. Solid line indicates the mean difference between the two measures, dashed lines indicate the limits of agreement (1.96 SDs of the mean difference).

people with stroke and traumatic brain injury, ${ }^{15}$ showing that step counts measured by the Fitbit tracker are highly correlated with step counts from other validated accelerometers and pedometers commonly used in research settings. Consistent with previous findings, ${ }^{10} 23$ the strong agreement and low absolute discrepancy ( $<10 \%$ error) between Fitbit and visually counted steps verifies the clinical utility of the Fitbit tracker for monitoring physical activity in older adults. The Fitbit tracker is relatively affordable, simple to use and allows self-monitoring and goal setting by providing feedback on the device itself or through the internet interface. ${ }^{24}$ The Fitbit's internet interface also stores long-term physical activity levels. As such, the feedback provided by the Fitbit may be used by older adults, individually or with guidance from a health professional, to encourage increased physical activity over time.

Our results demonstrated that ActiGraph accelerometers undercounted steps in older adults who had good overall mobility. This is evident in the lower proportion of participants whose Fitbit step counts were within 5-15\% of ActiGraph steps over a 7-day period in the presence of close percentage agreement between Fitbit and visually counted steps. The Bland-Altman plots also revealed that discrepancy between ActiGraph steps and both Fitbit and visually counted steps was exaggerated in individuals who took fewer steps during the 2MWT. These individuals were observed to have minor gait alteration, such as limping due to arthritic pain or peripheral neuropathy, and to walk more slowly. This finding is in agreement with previous findings demonstrating that accelerometers may not adequately identify steps or physical activity in people with gait alterations. ${ }^{16}$ 
The smaller leg accelerations generated during slower walking speeds ${ }^{25}$ in people with gait alterations are unlikely to trigger recognition of a step in accelerometers due to the high thresholds used to detect movement in these devices; ${ }^{26}$ however, this needs further investigation. ${ }^{27}$ It was also observed that many participants used pivot steps to turn during the 2MWT, particularly in corridors, which may explain the systematic undercounting of steps by the ActiGraph accelerometer over a short distance.

This study has established criterion validity of the Fitbit tracker for measuring physical activity in community-dwelling older adults. Yet the rapidity with which newer and cheaper pedometers are becoming available means that research validating these pedometers is unlikely to keep pace with the evolving technology, making it difficult for consumers and clinicians to determine the accuracy of each individual pedometer. ${ }^{5}$ Nevertheless, future studies are needed to confirm the effectiveness of physical activity monitors such as the Fitbit for increasing physical activity levels in older adults who do not currently meet physical activity recommendations; ${ }^{28}$ one such study is currently underway. ${ }^{20}$

Acknowledgements The authors wish to thank all the participants who participated. The authors also wish to thank Professor Chris Maher for his advice on statistical analysis.

Contributors AT and CS conceived the study. SSP, AT and CS initiated the study design. SSP, LMH, ER, CK and SC helped with implementation. SSP conducted the statistical analyses and drafted the manuscript. All authors reviewed the manuscript for critical intellectual content and all authors approved the final manuscript. SSP is responsible for the overall content of this manuscript as guarantor.

Funding This work is supported by a research bequest in addition to a Marrickville Council Community Grant and funding from the NSW Office of Communities, Sport and Recreation Participation and Facility Programme.

Competing interests CS receives salary funding from the National Health and Medical Research Council of Australia.

Ethics approval The Human Research Ethics Committee at The University of Sydney (approval number 2013/789). All participants provided written informed consent prior to data collection.

Provenance and peer review Not commissioned; externally peer reviewed.

Data sharing statement No additional data are available.

Open Access This is an Open Access article distributed in accordance with the Creative Commons Attribution Non Commercial (CC BY-NC 4.0) license, which permits others to distribute, remix, adapt, build upon this work noncommercially, and license their derivative works on different terms, provided the original work is properly cited and the use is non-commercial. See: http:// creativecommons.org/licenses/by-nc/4.0/

\section{REFERENCES}

1. Lee IM, Shiroma EJ, Lobelo F, et al. Effect of physical inactivity on major non-communicable diseases worldwide: an analysis of burden of disease and life expectancy. Lancet 2012;380:219-29.

2. Peeters GM, Mishra GD, Dobson AJ, et al. Health care costs associated with prolonged sitting and inactivity. Am J Prev Med 2014;46:265-72.

3. Department of Health. Australia's Physical Activity and Sedentary Behaviour Guidelines. 2014. http://www.health.gov.au/internet/main/ publishing.nsf/content/health-pubhlth-strateg-phys-act-guidelines
4. Loprinzi PD. Accelerometer-determined sedentary and physical activity estimates among older adults with diabetes: considerations by demographic and comorbidity characteristics. J Aging Phys Activ 2014;22:432-40.

5. Tudor-Locke C. Steps to better cardiovascular health: how many steps does it take to achieve good health and how confident are we in this number? Curr Cardiovasc Risk Rep 2010;4:271-6.

6. van der Ploeg HP, Chey T, Korda RJ, et al. Sitting time and all-cause mortality risk in 222497 Australian adults. Arch Intern Med 2012;172:494-500.

7. Ekblom-Bak E, Ekblom B, Vikström M, et al. The importance of non-exercise physical activity for cardiovascular health and longevity. Br J Sports Med 2014;48:233-8.

8. Tudor-Locke C, Johnson WD, Katzmarzyk PT. Relationship between accelerometer-determined steps/day and other accelerometer outputs in US adults. J Phys Act Health 2011;8:410-9.

9. Kang M, Marshall SJ, Barreira TV, et al. Effect of pedometer-based physical activity interventions: a meta-analysis. Res $Q$ Exerc Sport 2009;80:648-55.

10. Diaz KM, Krupka DJ, Chang MJ, et al. Fitbit(R): an accurate and reliable device for wireless physical activity tracking. Int $J$ Cardiol 2015;185:138-40.

11. Adam Noah J, Spierer DK, Gu J, et al. Comparison of steps and energy expenditure assessment in adults of Fitbit Tracker and Ultra to the Actical and indirect calorimetry. J Med Eng Technol 2013;37:456-62.

12. Tully MA, McBride C, Heron L, et al. The validation of Fibit Zip physical activity monitor as a measure of free-living physical activity. BMC Res Notes 2014;7:952.

13. Grant PM, Dall PM, Mitchell SL, et al. Activity-monitor accuracy in measuring step number and cadence in community-dwelling older adults. J Phys Act Health 2008;16:201-14.

14. Webber SC, Magill SM, Schafer JL, et al. GT3X+ accelerometer, Yamax pedometer and SC-StepMX pedometer step count accuracy in community-dwelling older adults. J Aging Phys Activ 2014;22:334-41.

15. Fulk GD, Combs SA, Danks KA, et al. Accuracy of 2 activity monitors in detecting steps in people with stroke and traumatic brain injury. Phys Ther 2014;94:222-9.

16. Taraldsen $\mathrm{K}$, Askim $\mathrm{T}$, Sletvold $\mathrm{O}$, et al. Evaluation of a body-worn sensor system to measure physical activity in older people with impaired function. Phys Ther 2011;91:277-85.

17. Trost SG, Tudor-Locke C. Advances in the science of objective physical activity monitoring: 3rd International Conference on Ambulatory Monitoring of Physical Activity and Movement. $\mathrm{Br} J$ Sports Med 2014;48:1009-10.

18. Mokkink LB, Terwee CB, Patrick DL, et al. COSMIN checklist manual. Amsterdam: EMGO Institute for Health and Care Research, VU University Medical Center, 2012.

19. Plasqui G, Westerterp KR. Physical activity assessment with accelerometers: an evaluation against doubly labeled water. Obesity Research (Silver Spring) 2007;15:2371-9.

20. Tiedemann A, Paul S, Ramsay E, et al. What is the effect of a combined physical activity and fall prevention intervention enhanced with health coaching and pedometers on older adults' physical activity levels and mobility-related goals? Study protocol for a randomised controlled trial. BMC Public Health 2015;15:477.

21. Lipton RB, Katz MJ, Kuslansky G, et al. Screening for dementia by telephone using the memory impairment screen. J Am Geriatr Soc 2003;51:1382-90.

22. Hallgren KA. Computing inter-rater reliability for observational data: an overview and tutorial. Tutor Quant Methods Psychol 2012;8:23-34.

23. Storm FA, Heller BW, Mazza C. Step detection and activity recognition accuracy of seven physical activity monitors. PLOS ONE 2015;10:e0118723.

24. Lyons EJ, Lewis ZH, Mayrsohn BG, et al. Behavior change techniques implemented in electronic lifestyle activity monitors: a systematic content analysis. J Med Internet Res 2014;16:e192.

25. Lelas JL, Merriman GJ, Riley PO, et al. Predicting peak kinematic and kinetic parameters from gait speed. Gait Posture 2003;17:106-12.

26. Tudor-Locke C, Ainsworth BE, Thompson RW, et al. Comparison of pedometer and accelerometer measures of free-living physical activity. Med Sci Sport Exer 2002;34:2045-51.

27. Lee IM, Shiroma EJ. Using accelerometers to measure physical activity in large-scale epidemiological studies: issues and challenges. Br J Sports Med 2014;48:197-201.

28. Trost SG, O'Neil M. Clinical use of objective measures of physical activity. Br J Sports Med 2014;48:178-81. 